\title{
Beyond Abdominal Pain: Pain Beliefs, Pain Affect, and Distress as Determinants of Quality of Life in Patients with Chronic Pancreatitis
}

\author{
Craig E. Keller, M.D. ${ }^{1}$, C. Mel Wilcox, M.D. ${ }^{2}$, Gregory D. Gudleski, Ph.D. ${ }^{1}$, Stacey Branham², \\ and Jeffrey M. Lackner, Psy.D. ${ }^{1}$ \\ ${ }^{1}$ Department of Medicine, Jacobs School of Medicine and Biomedical Sciences, SUNY, Buffalo, \\ NY, United States \\ ${ }^{2}$ Department of Medicine, University of Alabama at Birmingham, Birmingham, AL, United States
}

\section{Abstract}

Goals-To assess the relationship between pain, psychological processes and quality of life in chronic pancreatitis.

\begin{abstract}
Background-Chronic pancreatitis is a progressive inflammatory disorder of the pancreas characteristically resulting in abdominal pain and impairing quality of life. Pain due to chronic pancreatitis is poorly understood and frequently difficult to treat. This pain has historically been understood as a peripheral process originating from the pancreas itself, but a growing body of literature is revealing an important role offered by central influences. Viewed through the perspective of the biopsychosocial model of illness, cognitive variables strongly influence quality of life. However, there is little understanding of variables that influence quality of life in chronic pancreatitis.
\end{abstract}

Study-Patients with chronic pancreatitis from the University of Alabama at Birmingham were administered a 165-question test battery which was comprised of questionnaires evaluating pain beliefs, disease-specific quality of life, psychological distress, pain sensation, pain affect, and long-term suffering.

Results-Sixty-eight subjects completed the question battery between 2/28/2011 and 1/16/2014 . Almost all (91.2\%) reported taking pain medication. Quality of life was significantly associated with reported levels of pain intensity $(r=-.52, p<.01)$ as well as perceived self-blame.

Conclusions-The significant predictors of quality of life impairment in chronic pancreatitis are pain intensity and perceived self-blame for pain. Further research is needed to elucidate this relationship while also evaluating the effectiveness of systematic modification of these variables in an attempt to improve pain and quality of life in chronic pancreatitis.

Correspondence: Craig Keller, University at Buffalo, Department of Medicine, Behavioral Medicine Clinic, Erie County Medical Center, 462 Grider Street, Buffalo, NY 14215, Tel: (716) 898-6254, Fax: (716) 898-3040, craig.e.keller@ gmail.com. 


\section{Introduction}

Chronic pancreatitis (CP) is a progressive inflammatory disorder of the pancreas characteristically resulting in abdominal pain. What often begins as time-limited episodes of acute abdominal pain frequently progresses to more persistent episodes accompanied by endocrine and exocrine pancreatic insufficiency. The character and severity of pain are quite variable but in some patients it may be severe, intractable, and result in significant impairment in quality of life. With progression of disease, endocrine and exocrine insufficiency may result further impairing daily activities and long-term outcome. Pain due to chronic pancreatitis is not well understood, multifactorial, and often times difficult to treat 1 . For patients with CP, pain is the most troublesome symptom and the one for which they most often seek medical attention. For providers, it is also the symptom by which the effectiveness of treatment is measured. In spite of its clinical importance, CP pain is neither well-understood nor satisfactorily treated.

In general, $\mathrm{CP}$ pain has historically been understood as the product of nociceptive stimulation from peripheral input caused by tissue damage (i.e., pancreatic ductal hypertension, tissue ischemia, perineural inflammation, etc.). However, evidence suggests pain in chronic pancreatitis is not solely a result of organ-specific, anatomic disturbances. A recent study failed to find a correlation between abdominal imaging features and pain patterns in chronic pancreatitis ${ }^{2}$. There are empirical and conceptual reasons to believe that CP pain is not strictly a symptom of underlying peripheral disease activity. After all, 10-15\% of CP patients do not have any pain at all $^{3}$, and some patients continue to experience abdominal pain even after complete removal of their pancreas ${ }^{4}$. These findings suggest that $\mathrm{CP}$ pain is not simply a peripherally-mediated phenomenon but one subject to central influences. A growing body of literature highlights the importance of central influences in chronic pancreatitis (i.e., central sensitization and impairments in inhibitory pain modulation, etc. $)^{5,6}$.

One potentially useful way of understanding complex chronic pain syndromes comes from the biopsychosocial model of illness ${ }^{7}$. This model would view CP pain as a complex phenomenon involving biological, psychological, and social mechanisms, all of which interact and are reciprocally related. Pain therefore is not only influenced by biobehavioral factors but it also produces biological, psychological, and social changes that in turn affect future responses to pain. Of psychological factors, cognitive factors (such as patients' thoughts, beliefs, expectations and coping behaviors) exert a particularly strong influence on the perception, reporting and response to pain ${ }^{8-10}$. Beliefs patients have about pain are a powerful cognitive variable that strongly influences quality of life (QOL), which is impaired in CP. While CP is associated with impaired QOL, there is little understanding of variables that influence QOL. The methods used to assess quality of life in CP have been poorly studied and the most important factors, both cognitive and behavioral, affecting QOL remain little studied.

The purpose of this study was to assess the relationship between pain, psychological processes and QOL, with a focus on cognitive processes that have been identified as critical 
in other chronic painful medical disorders. Given QOL impairment among CP patients, we would expect that their pain beliefs would impair their function and well-being.

\section{Materials and Methods}

\section{Participants}

Subjects included 68 chronic pancreatitis patients who were referred to a specialty pancreatic clinic at the University of Alabama at Birmingham, a tertiary care hospital and a major referral center for the state of Alabama. Informed consent was obtained from each subject. The study was approved by the Institutional Review Boards of both the University at Buffalo and University of Alabama at Birmingham.

\section{Definitions}

All patients were prospectively evaluated by a principal investigator (CMW). The diagnosis of chronic pancreatitis was established by the presence of typical history, computed tomographic findings characteristic of chronic pancreatitis including pancreatic calcifications and/or endoscopic ultrasonography demonstrating five or more features of chronic pancreatitis, or by evaluation of pathological tissue removed at the time of any prior pancreatic surgery. The presence of other complications of chronic pancreatitis including diabetes and exocrine insufficiency were recorded at the time of evaluation. Some patients evaluated had complications of chronic pancreatitis including pseudocyst.

Data collection was performed from 2/28/2011 to 1/16/2014. Each patient was seen by one of the investigators (CMW) where a complete history and physical examination was performed and any pertinent radiographs reviewed.

\section{Questionnaire}

Subjects were administered a 165-question test battery that for the purpose of this study included five psychometrically validated questionnaires.

\section{Pain Beliefs and Perceptions}

The Pain Beliefs and Perceptions Inventory (PBPI) is a 16-question scale addressing 4 dimensions of pain beliefs: seeing pain as mysterious (Mystery), holding oneself responsible for pain (Self-Blame), regarding one's condition as lingering into the future (Permanence), and/or continuous over time (Constancy). When used in heterogeneous samples of patients suffering from chronic somatic and visceral pain, beliefs that pain would be enduring and constant were associated with increased reported pain and intensity ${ }^{10}$. High scores in Permanence and Mystery scales have been correlated with more catastrophizing and worse coping and disability ${ }^{11-13}$. A correlation has also been found between beliefs of permanence, mystery, self-blame, and psychological distress (anxiety and depressive symptoms $)^{10}$.

\section{Disease-Specific Quality of Life}

The European Organization for Research and Treatment of Cancer QLQ-C30 (EORTC QLQ-C30) is a 30 item questionnaire originally developed for the evaluation of quality of 
life in patients affected with pancreatic cancer ${ }^{14}$. The QLQ-C30 (version 1) incorporated five functional scales (physical, role, cognitive, emotional, and social), three symptom scales (fatigue, pain, and nausea and vomiting), a global health status/QOL scale, and a number of single items assessing additional symptoms commonly reported by cancer patients (i.e., dyspnea, loss of appetite, insomnia, constipation and diarrhea) and perceived financial impact of the disease. Subsequent versions were built upon the same basic principles, culminating in the 'core' 30-item EORTC QLQ-C30 (version 3.0) questionnaire, representing over 20 years of continuous development, refinement and validation. Individuals are instructed to rate on a scale of 1 ("Not at all") to 4 ("Very Much") the extent to which their disease affects various aspects of their physical, mental, and social well-being.

Given that many of the presenting symptoms and palliative treatment options available to patients with pancreatic cancer are similar to those available for chronic pancreatitis, attention has focused on the applicability of this questionnaire to patients with $\mathrm{CP}$. Psychometric evaluation has revealed strong content validity, internal consistency, convergent and discriminant validity ${ }^{15}$, lending further support to its use in chronic pancreatitis.

\section{Psychological Distress}

The Depression Anxiety Stress Scale (DASS 21) is a 21 item self-report questionnaire designed to measure the severity of a range of symptoms common to both Depression and Anxiety. In completing the DASS, the individual indicates the presence of a symptom over the previous week. Each item is scored numerically from 0 (did not apply to me at all over the last week) to 3 (applied to me very much or most of the time over the past week). The essential function of the DASS is to assess the severity of the core symptoms of Depression, Anxiety and Stress. Accordingly, the DASS allows not only a way to measure the severity of a patient's symptoms but a means by which a patient's response to treatment can also be measured $^{16,17}$.

\section{Pain Sensation and Pain Affect}

Immediate pain affect (pain sensation, or cognitive appraisal of threat) was assessed using the short form of the McGill Pain Questionnaire (SF-MPQ) ${ }^{18}$. The SF-MPQ consists of 15 words reflecting the most commonly used adjectives for describing the sensory (11 words) and affective (4 words) quality of pain experience during four weeks prior to assessment. Patients rate the intensity of these descriptors on a four-point scale (where $0=$ none, $1=$ mild, $2=$ moderate, $3=$ severe). Psychometric studies have found strong correlations between the major indices of the SF-MPQ and the original version ${ }^{19}$ which previous pain processing research has used to measure pain sensation and pain affect ${ }^{20}$.

\section{Long-Term Suffering}

Secondary pain affect (long term suffering or meaning of pain experience) was measured using the Pain Discomfort Scale (PDS) ${ }^{21}$. The PDS is a 10-item measure that requires the subjects to rate on a 5-point scale ranging from 0 (this is very untrue for me) to 4 (this is very true for me) the extent to which they agree with cognitive and affective responses associated with pain-related suffering (e.g., "the pain I experience is unbearable"). The PDS 
has sound psychometric properties (internal consistently, test-retest reliability, construct validity) ${ }^{21}$.

Pain affect is a dimensional construct moderated by cognitive appraisal of threat (immediate pain affect) or long-term meaning of pain experience (secondary pain affect) ${ }^{22,23}$.

\section{Data analysis plan}

Descriptive statistics (e.g., means, SD, and percentages) were used to summarize demographic and clinical data. Partial correlations were then used to examine the bivariate relationships among the variables while controlling for possible confounding variables.

Finally, a hierarchical regression analysis was performed to determine significant independent predictors of QOL while controlling for the effects of other variables. Conceptually distinct blocks of independent variables (i.e., pain, psychological distress, pain beliefs) were entered sequentially with pain variables entered first, followed by psychological distress and pain beliefs variables. In order to limit the number of variables in the regression models, only variables with a p-value of less than .01 in the bivariate analyses were entered into the equations. All data analyses were performed using SPSS 23.0 (SPSS, Chicago, IL).

\section{Results \\ Descriptive analyses}

Table 1 shows the demographic and basic clinical characteristics of the sample. The sample was mostly middle-aged, male, Caucasian, and in a current relationship. Most of the sample had a high school education or less with a middle income. Fifty-five percent of the sample reported suffering from pancreatitis pain for 5 years or more. The average QOL score on the EORTC QLQ-C30 of 37.5 was significantly lower than mean reference scores for the general population (71.2) and for patients with all stages of liver/bile/pancreas cancer $(55.9)^{24}$. Almost all (91.2\%) reported taking some kind of pain medication, with strong opiates $(64.7 \%)$ and acetaminophen $(27.9 \%)$ being the most common. Only $25.0 \%$ of the sample was working full-time and $44.1 \%$ were on disability. Nearly half (48.5\%) identified as a current cigarette smoker and $72.1 \%$ reported that they had smoked during their lifetime. Only about one-third of the sample (35.3\%) reported drinking alcohol in the past 12 months and $5.9 \%$ reported having 5 or more drinks on a typical day when drinking. The two most common medical comorbidities were hypertension (50.0\%) and diabetes (29.4\%).

\section{Bivariate analyses}

Partial correlations were conducted to assess the magnitude of the relations between QOL and the pain and psychological variables while controlling for possible confounding factors including age, gender, education level, and duration of illness. As seen in Table 2, the results were in the expected manner. With respect to pain variables, QOL was significantly associated with a patient's reported average levels of pain and the sensory and affect subscales of the McGill questionnaire. Specifically, patients reported greater QOL impairment as the intensity, sensory experience, and emotional unpleasantness of pain increased. The strength of the associations was greatest for the average level of the intensity 
of pain $(\mathrm{r}=-.52, \mathrm{p}<.01)$. The correlation between QOL and pain discomfort (i.e., secondary pain affect) did not reach statistical significance.

Regarding psychological variables, QOL was significantly associated with 3 of the 4 pain beliefs dimensions. CP patients with lower QOL scores tended to blame themselves for pain, see pain as more mysterious, and characterize the duration of their pain episodes as more continuous. The strength of the correlations was similar across all 3 dimensions.

Permanence was the only pain belief dimension that did not significantly correlate with QOL. With respect to psychological distress, QOL impairment was significantly associated with greater reported levels of stress and depression. However, the correlation between QOL and anxiety was not statistically significant.

\section{Regressions analyses}

A hierarchical regression analysis was performed in order to examine the unique contributions of the pain and psychological variables in the explanation of QOL. As stated previously, only the variables that had a p-value of less than .01 in the bivariate analyses were entered into the models. Furthermore, none of the demographic or potential confounding variables that were controlled for in the bivariate analyses were significantly associated with the QOL measure and were not included in the regression models. Due to the high intercorrelations among some of the predictor variables in the regression model, we first conducted tests for multicollinearity. The results showed that variance inflation factors ranged from 1.05 to 3.78 suggesting that the standard errors of the coefficients were not inflated by multicollinearity. Pain variables were entered into the regression equation in the first step; psychological distress variables were entered in the second step; and pain beliefs variables were introduced in the third step.

The results of the regression analyses are shown in Table 3. In step 1, pain variables explained $40.8 \%$ of the variance in QOL $(\mathrm{F}=12.8, \mathrm{p}<.001)$. Level of pain intensity was the only statistically significant independent variable. In step 2, the psychological distress variables explained an additional 3.0\% of the variance in QOL, however this increase was not statistically significant $(\Delta \mathrm{F}=1.49, \mathrm{p}=.24)$. Level of pain intensity continued to be the only statistically significant predictor at this step. Finally, step 3 introduced the pain beliefs variables which explained an additional $10.6 \%$ of the variance in $\mathrm{QOL}(\Delta \mathrm{F}=3.32, \mathrm{p}=.02)$. The final model explained $54.4 \%$ of the variance in QOL scores $(7.45, \mathrm{p}<.001)$, with level of pain intensity and the pain beliefs dimension of self-blame being the only statistically significant independent predictors.

\section{Discussion}

Chronic pancreatitis is a progressive disease characterized by impaired quality of life and oftentimes debilitating abdominal pain. While a number of researchers have studied QOL in chronic pancreatitis ${ }^{25-28}$, none has assessed the extent to which QOL impairment is due to patients' beliefs of pain. In this study, we found a correlation between pain beliefs and QOL impairment such that more patients with more negatively skewed beliefs had worse QOL. Patients with worse QOL tended to blame themselves for pain, see pain as more mysterious, and characterize their pain as more continuous in nature. These data argue against the notion 
that QOL is due to solely to pain intensity or the manifestations of CP such as the effects of exocrine and endocrine pancreatic insufficiency. This is not to say that pain dimensions such as the sensory quality of pain (e.g. intensity) are unimportant. Indeed, we found that QOL was associated with both the intensity of pain and its emotional unpleasantness. Further research is needed to assess the relative impact of different aspects of pain on health outcomes. The broader research shows that the affective aspect of pain plays an increasingly important role on the trajectory of pain over time ${ }^{29}$.

The finding that cognitive factors are associated with pain is consistent with research highlighting the role of central processes in altering the perception, processing, and response to painful stimuli as well as in chronic pancreatitis ${ }^{30-36}$. These studies have shown that patients with chronic pancreatitis have alterations in central pain processing similar to those seen in other chronic pain disorders (i.e., sensitization, cortical reorganization, and alterations in endogenous pain modulation), while others have demonstrated the beneficial effects of pharmacotherapy aimed at targeting those central variables. For example, Bouwense and colleagues ${ }^{5}$ demonstrated an anti-hyperalgesic effect of pregabalin, a centrally-acting alpha-2 receptor agonist which is commonly-used for its neuro-inhibitory effects within the central nervous system, while others have demonstrated an antinociceptive effect of pregabalin in patients with chronic pancreatitis 6 .

Given our current knowledge regarding the complex pathogenesis of pain, it is important to systematically assess the relative contribution of peripheral and central factors to the contribution of pain in patients with chronic pancreatitis. While the central nervous system has been investigated with respect to its processing and modification of neurotransmission in chronic pancreatitis, no study to date has objectively investigated changes in the central nervous system as they relate to cognitive processes in this disease. The present study identifies relationships among cognitive variables and pain in chronic pancreatitis. Future research could further advance our understanding of this relationship through the utilization of neuroimaging (i.e., functional MRI, PET scans, evoked brain potentials, etc.) to investigate potential structural and functional changes within the CNS as they relate to these cognitive processes and the treatment thereof.

These data suggest that pain of chronic pancreatitis is complex, multidimensional, and not solely a product of nociceptive stimulation from the pancreas. Instead, pain in chronic pancreatitis is influenced by the interplay of a host of biological, psychological, and environmental factors. It is important for the clinician to consider this complex relationship when conventional treatment regimens focusing on peripheral processes alone fall short of treatment expectations for patients whose pain is subject to strong central factors. Further research is needed to evaluate the utility of systematic psychological modification of faulty cognitive processes, and its effect on quality of life and the overall pain experience in chronic pancreatitis.

There are several strengths of this study. To our knowledge, this is the first study evaluating the relationship of cognitive factors with pain perception and quality of life impairment in chronic pancreatitis. Quality of life in chronic pancreatitis can be evaluated with wellstudied generic questionnaires or a recently-developed disease-specific questionnaire (the 
Pancreatitis Quality of Life Instrument), which has been psychometrically evaluated for use in chronic pancreatitis patients ${ }^{37}$. The use of several psychometrically-validated questionnaires in our survey allows the evaluation of numerous cognitive and physical variables in this disease. The relatively high response rate allows minimization of participation bias. The limitations of our study deserve mention. The study sample is relatively small, and was recruited from a single institution. This may limit the generalizability of our findings. The lack of a control group makes it difficult to know whether our findings are specific to chronic pancreatitis or characteristic of patients with other painful GI disorders. While our assessment battery featured a novel set of instruments evaluating aspects of the pain experience that have not been applied to CP patients, the battery was not designed to tap into the universe of biobehavioral processes that influence pain perception in this population. Also, there may have been other factors which were not evaluated which could have impacted our findings.

That said, our study demonstrates the relationship of cognitive factors, the pain experience, and quality of life impairment in individuals affected with chronic pancreatitis. Further research is needed to elucidate this relationship while also evaluating the effectiveness of systematic modification of these variables in an attempt to improve pain and quality of life in chronic pancreatitis.

\section{Acknowledgments}

This study is supported partly by NIH grant DK77738.

\section{References}

1. Anderson MA, Akshintala V, Albers KM, et al. Mechanism, assessment and management of pain in chronic pancreatitis: Recommendations of a multidisciplinary study group. Pancreatology. 2016; 16:83-94. [PubMed: 26620965]

2. Wilcox CM, Yadav D, Ye T, et al. Chronic pancreatitis pain pattern and severity are independent of abdominal imaging findings. Clin Gastroenterol Hepatol. 2015; 13:552-60. quiz e28-9. [PubMed: 25424572]

3. Shafiq N, Rana S, Bhasin D, et al. Pancreatic enzymes for chronic pancreatitis. Cochrane Database Syst Rev. 2009 Cd006302.

4. Lieb JG 2nd, Forsmark CE. Review article: pain and chronic pancreatitis. Aliment Pharmacol Ther. 2009; 29:706-19. [PubMed: 19284407]

5. Bouwense SA, Olesen SS, Drewes AM, et al. Pregabalin and placebo responders show different effects on central pain processing in chronic pancreatitis patients. J Pain Res. 2015; 8:375-86. [PubMed: 26203273]

6. Olesen SS, Graversen C, Olesen AE, et al. Randomised clinical trial: pregabalin attenuates experimental visceral pain through sub-cortical mechanisms in patients with painful chronic pancreatitis. Aliment Pharmacol Ther. 2011; 34:878-87. [PubMed: 21848870]

7. Engel GL. The need for a new medical model: a challenge for biomedicine. Science. 1977; 196:129-36. [PubMed: 847460]

8. Grover M, Drossman DA. Functional abdominal pain. Curr Gastroenterol Rep. 2010; 12:391-8. [PubMed: 20694840]

9. Turk DC, Rudy TE. Assessment of cognitive factors in chronic pain: a worthwhile enterprise? J Consult Clin Psychol. 1986; 54:760-8. [PubMed: 3540044]

10. Williams DA, Robinson ME, Geisser ME. Pain beliefs: assessment and utility. Pain. 1994; 59:718. [PubMed: 7854805] 
11. Herda CA, Siegeris K, Basler HD. The Pain Beliefs and Perceptions Inventory: further evidence for a 4-factor structure. Pain. 1994; 57:85-90. [PubMed: 8065801]

12. Walsh DA, Radcliffe JC. Pain beliefs and perceived physical disability of patients with chronic low back pain. Pain. 2002; 97:23-31. [PubMed: 12031776]

13. Williams DA, Keefe FJ. Pain beliefs and the use of cognitive-behavioral coping strategies. Pain. 1991; 46:185-90. [PubMed: 1749641]

14. Aaronson NK, Ahmedzai S, Bergman B, et al. The European Organization for Research and Treatment of Cancer QLQ-C30: a quality-of-life instrument for use in international clinical trials in oncology. J Natl Cancer Inst. 1993; 85:365-76. [PubMed: 8433390]

15. Fitzsimmons D, Kahl S, Butturini G, et al. Symptoms and quality of life in chronic pancreatitis assessed by structured interview and the EORTC QLQ-C30 and QLQ-PAN26. Am J Gastroenterol. 2005; 100:918-26. [PubMed: 15784041]

16. Foundation. SHLPFLP. Manual for the depression, anxiety, stress scales. Sydney: Psychology Foundation; 1995.

17. Gomez R, Summers M, Summers A, et al. Depression Anxiety Stress Scales-21: measurement and structural invariance across ratings of men and women. Assessment. 2014; 21:418-26. [PubMed: 24486523]

18. Melzack R. The short-form McGill Pain Questionnaire. Pain. 1987; 30:191-7. [PubMed: 3670870]

19. Melzack R. The McGill Pain Questionnaire: major properties and scoring methods. Pain. 1975; 1:277-99. [PubMed: 1235985]

20. Harkins SW, Price DD, Braith J. Effects of extraversion and neuroticism on experimental pain, clinical pain, and illness behavior. Pain. 1989; 36:209-18. [PubMed: 2919101]

21. Jensen MP, Karoly P, Harris P. Assessing the affective component of chronic pain: development of the Pain Discomfort Scale. J Psychosom Res. 1991; 35:149-54. [PubMed: 2046049]

22. Lackner JM, Jaccard J, Blanchard EB. Testing the sequential model of pain processing in irritable bowel syndrome: a structural equation modeling analysis. Eur J Pain. 2005; 9:207-18. [PubMed: 15737813]

23. Price, DD. Psychological and Neural Mechanisms of Pain. New York: Raven Press; 1988.

24. Scott NWF, P M, Aaronson NK, Bottomley A, De Graeff A, Groenvold M, Gundy C, Koller M, Peterson MA, AG Sprangers M. EORTC QLQ-C30 Reference Values. 2008:154.

25. Mokrowiecka A, Pinkowski D, Malecka-Panas E. Assessment of quality of life in patients with chronic pancreatitis. Med Sci Monit. 2011; 17:Cr583-8. [PubMed: 21959613]

26. Mullady DK, Yadav D, Amann ST, et al. Type of pain, pain-associated complications, quality of life, disability and resource utilisation in chronic pancreatitis: a prospective cohort study. Gut. 2011; 60:77-84. [PubMed: 21148579]

27. Pezzilli R, Morselli-Labate AM, Fantini L, et al. Assessment of the quality of life in chronic pancreatitis using Sf-12 and EORTC Qlq-C30 questionnaires. Dig Liver Dis. 2007; 39:1077-86. [PubMed: 17692582]

28. Wehler M, Reulbach U, Nichterlein R, et al. Health-related quality of life in chronic pancreatitis: a psychometric assessment. Scand J Gastroenterol. 2003; 38:1083-9. [PubMed: 14621285]

29. Chapman CR, Gavrin J. Suffering: the contributions of persistent pain. Lancet. 1999; 353:2233-7. [PubMed: 10393002]

30. Bouwense SA, Olesen SS, Drewes AM, et al. Is altered central pain processing related to disease stage in chronic pancreatitis patients with pain? An exploratory study PLoS One. 2013; 8:e55460. [PubMed: 23405154]

31. Demir IE, Tieftrunk E, Maak M, et al. Pain mechanisms in chronic pancreatitis: of a master and his fire. Langenbecks Arch Surg. 2011; 396:151-60. [PubMed: 21153480]

32. Drewes AM, Krarup AL, Detlefsen S, et al. Pain in chronic pancreatitis: the role of neuropathic pain mechanisms. Gut. 2008; 57:1616-27. [PubMed: 18566105]

33. Lelic D, Olesen SS, Hansen TM, et al. Functional reorganization of brain networks in patients with painful chronic pancreatitis. Eur J Pain. 2014; 18:968-77. [PubMed: 24402765]

34. Bouwense SA, Ahmed Ali U, ten Broek RP, et al. Altered central pain processing after pancreatic surgery for chronic pancreatitis. Br J Surg. 2013; 100:1797-804. [PubMed: 24227367] 
35. Dimcevski G, Sami SA, Funch-Jensen P, et al. Pain in chronic pancreatitis: the role of reorganization in the central nervous system. Gastroenterology. 2007; 132:1546-56. [PubMed: 17408654]

36. Frokjaer JB, Bouwense SA, Olesen SS, et al. Reduced cortical thickness of brain areas involved in pain processing in patients with chronic pancreatitis. Clin Gastroenterol Hepatol. 2012; 10:4348.e1. [PubMed: 22155560]

37. Wassef W, DeWitt J, McGreevy K, et al. Pancreatitis Quality of Life Instrument: A Psychometric Evaluation. Am J Gastroenterol. 2016; 111:1177-86. [PubMed: 27296943] 
Table 1

Demographics and clinical characteristics $(N=68)$

\begin{tabular}{|c|c|c|c|c|}
\hline & $\mathbf{n}$ & $\%$ & Mean & SD \\
\hline Age (years) & & & 49.2 & 11.3 \\
\hline Gender (Male) & 42 & $61.8 \%$ & & \\
\hline \multicolumn{5}{|l|}{ Relationship Status } \\
\hline Married/Life Partner & 39 & $57.4 \%$ & & \\
\hline Divorced & 8 & $11.8 \%$ & & \\
\hline Widowed & 3 & $4.4 \%$ & & \\
\hline Separated & 5 & $7.3 \%$ & & \\
\hline Single & 8 & $11.8 \%$ & & \\
\hline Cohabitating & 5 & $7.3 \%$ & & \\
\hline \multicolumn{5}{|l|}{ Race } \\
\hline American Indian & 4 & $5.9 \%$ & & \\
\hline African American & 15 & $22.1 \%$ & & \\
\hline Caucasian & 49 & $72.0 \%$ & & \\
\hline \multicolumn{5}{|l|}{ Education } \\
\hline High school or less & 47 & $69.1 \%$ & & \\
\hline College degree & 15 & $22.1 \%$ & & \\
\hline Post-graduate & 6 & $8.8 \%$ & & \\
\hline Income (thousands of dollars) & & & 45.8 & 32.7 \\
\hline Duration of symptoms (years) & & & 9.2 & 6.8 \\
\hline Average pain past week & & & 5.8 & 2.6 \\
\hline \multicolumn{5}{|l|}{ Pain medications } \\
\hline Acetaminophen & 19 & $27.9 \%$ & & \\
\hline Aspirin & 5 & $7.4 \%$ & & \\
\hline Nonsteroidal anti-inflammatory & 8 & $11.8 \%$ & & \\
\hline Cox II inhibitors & 1 & $1.5 \%$ & & \\
\hline Tramadol & 10 & $14.7 \%$ & & \\
\hline Mild opiates 1 & 1 & $1.5 \%$ & & \\
\hline Stronger opiates ${ }^{2}$ & 44 & $64.7 \%$ & & \\
\hline \multicolumn{5}{|l|}{ Medical comorbidities } \\
\hline Hypertension & 34 & $50.0 \%$ & & \\
\hline Heart disease & 11 & $16.2 \%$ & & \\
\hline Stroke & 2 & $2.9 \%$ & & \\
\hline Diabetes & 20 & $29.4 \%$ & & \\
\hline Cirrhosis of liver & 3 & $4.4 \%$ & & \\
\hline Chronic lung disease & 3 & $4.4 \%$ & & \\
\hline Fibromyalgia & 5 & $7.4 \%$ & & \\
\hline Osteoporosis & 9 & $13.2 \%$ & & \\
\hline Endometriosis & 2 & $2.9 \%$ & & \\
\hline Quality of Life & & & 37.5 & 23.5 \\
\hline
\end{tabular}

J Clin Gastroenterol. Author manuscript; available in PMC 2019 July 01. 
Note.

${ }^{1}$ Fiorinal, Fiorcet, Darvon, Darvocet;

2 Codeine, hydrocodone, oxycodone, Demerol, morphine, methadone, Duragesic patch. 


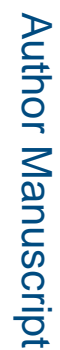

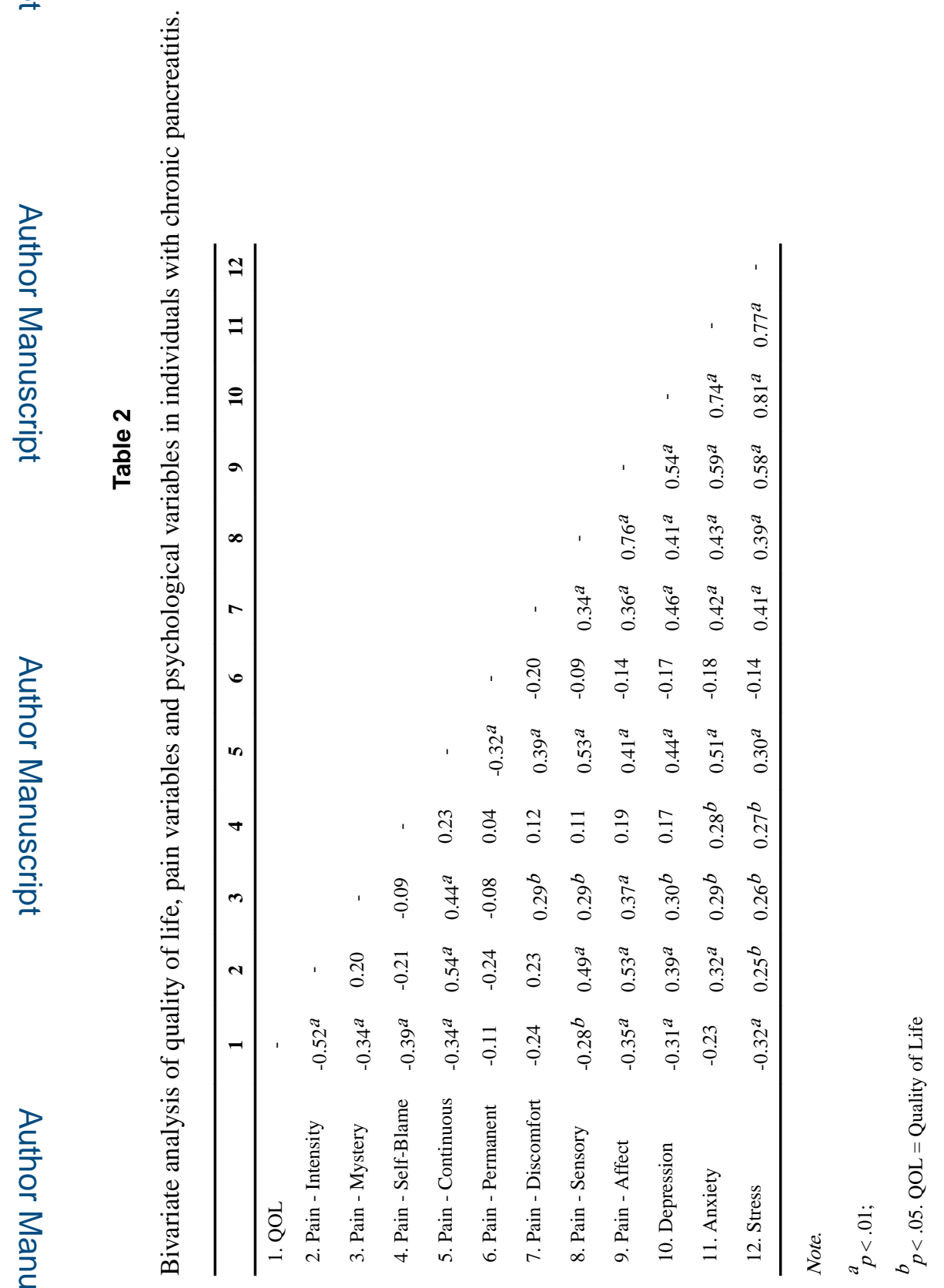

J Clin Gastroenterol. Author manuscript; available in PMC 2019 July 01. 

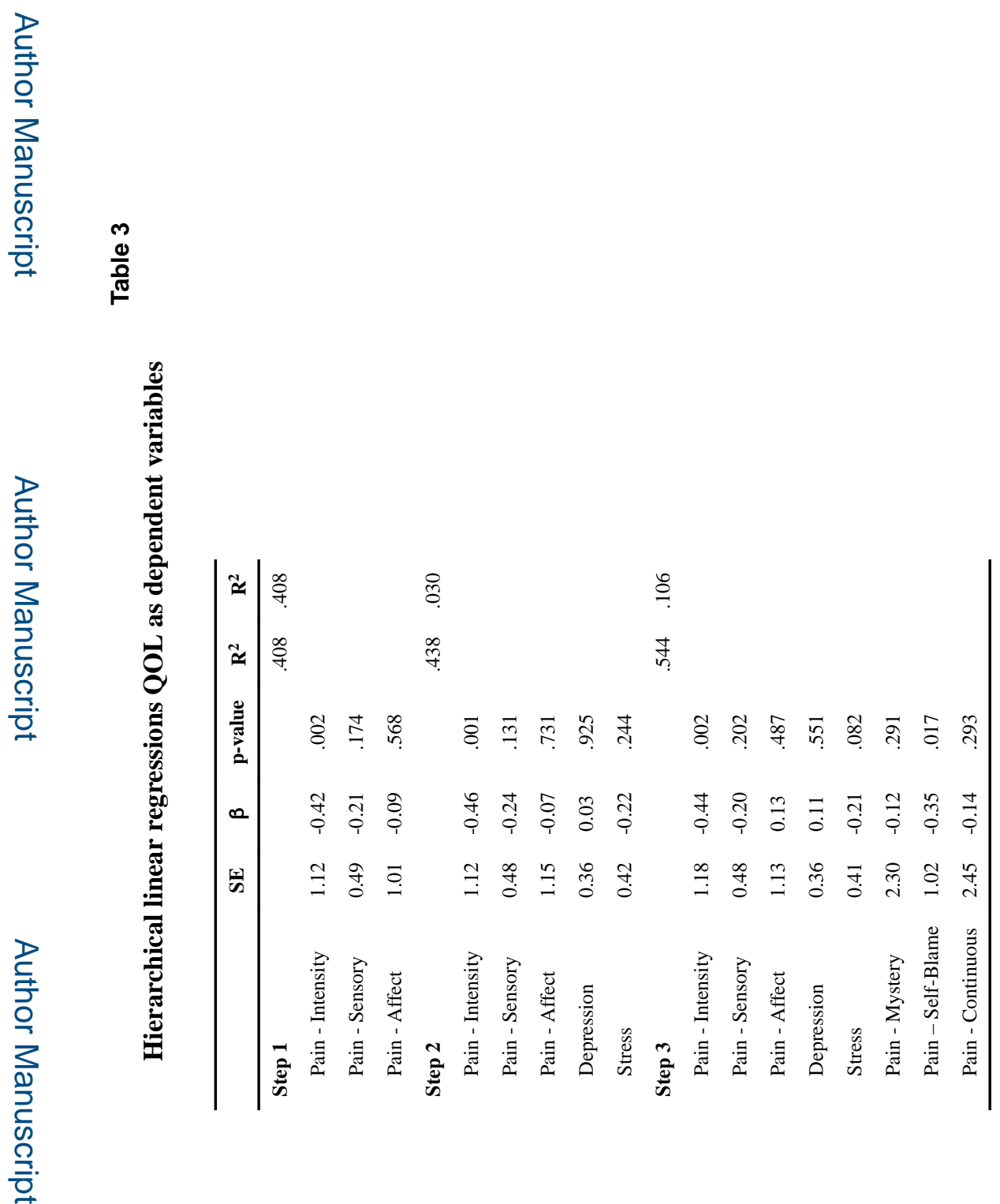